\title{
Quantum and classical correlations inside the entanglement wedge
}

\author{
Koji Umemoto॰ \\ Center for Gravitational Physics, Yukawa Institute for Theoretical Physics, Kyoto University, \\ Kitashirakawa Oiwakecho, Sakyo-ku, Kyoto 606-8502, Japan
}

(Received 27 August 2019; published 20 December 2019)

\begin{abstract}
We show that the entanglement wedge cross section (EWCS) can become larger than the quantum entanglement measures such as the entanglement of formation in the AdS/CFT correspondence. We then discuss a series of holographic duals to the optimized correlation measures, finding a novel geometrical measure of correlation, the entanglement wedge mutual information (EWMI), as the dual of the $Q$ correlation. We prove that the EWMI satisfies the properties of the $Q$-correlation as well as the strong superadditivity, and that it can become larger than the entanglement measures. These results imply that both of the EWCS and the EWMI capture more than quantum entanglement in the entanglement wedge, which enlightens a potential role of classical correlations in holography.
\end{abstract}

DOI: 10.1103/PhysRevD.100.126021

\section{INTRODUCTION}

Quantum entanglement has provided a key tool to study various aspects of modern physics from condensed matter theory to the black hole evaporation. In the AdS/CFT correspondence [1-3], quantum entanglement also plays a central role in the investigation of how the bulk geometrical data are encoded in the boundary field theory [4-8]. The Ryu-Takayanagi (RT) formula $[9,10]$ (or the HubenyRangamani-Takayanagi (HRT) formula [11,12] for covariant cases) tells us that the von Neumann entropy associated with a spacial subregion $A$ in CFTs $S_{A} \equiv S\left(\rho_{A}\right)=$ $-\operatorname{Tr} \rho_{A} \log \rho_{A}$ is equivalent to the area of codimension-2 minimal surface $\gamma_{A}$ that is anchored on the entangling surface $\partial A$ and homologous to $A$,

$$
S_{A}=\min _{\gamma_{A}} \frac{\operatorname{Area}\left(\gamma_{A}\right)}{4 G_{N}},
$$

at the leading order of the large $N$ limit. The von Neumann entropy $S_{A}$ is commonly called the entanglement entropy (EE) because this quantifies an amount of quantum entanglement between $A$ and its complement $A^{c}$ when the total state is pure [13]. For mixed states, however, the von Neumann entropy no longer deserves to be a measure of correlation, and thus we need to find another geometrical way to measure correlations.

A generalization of the Ryu-Takayanagi surface, the entanglement wedge cross section (EWCS), was introduced

Published by the American Physical Society under the terms of the Creative Commons Attribution 4.0 International license. Further distribution of this work must maintain attribution to the author(s) and the published article's title, journal citation, and DOI. Funded by SCOAP ${ }^{3}$. in $[14,15]$ as the minimal cross section of the entanglement wedge [16-18]. This is a geometrical measure of correlations between the boundary subsystems connected by the entanglement wedge that are usually in mixed states. Thus the EWCS in boundary theories is expected to be dual to some correlation measure that is a generalization of $\mathrm{EE}$ for mixed states.

The EWCS was originally conjectured to be the dual of the entanglement of purification (EOP) [19], based on agreements of their various information-theoretic properties $[14,15]$ as well as compatibility with the tensor network description of AdS/CFT [20,21]. The proposal has passed further consistency checks in the multipartite generalization [22] and in the conditional generalization [23,24]. Refer to [25-40] for recent progress.

Surprisingly, several correlation measures other than EOP have been shown to be essentially equal to the EWCS with appropriate coefficients, including the logarithmic negativity [41-44], the odd entropy [45], and the reflected entropy [46]. With the monogamy of holographic mutual information [47] in mind, which strongly suggests that quantum entanglement dominates holographic correlations, we may speculate that some axiomatic measure of quantum entanglement (see, e.g., [48]) would also be equivalent to the EWCS in holographic CFTs.

In this paper, however, we present a no-go theorem in this direction: the EWCS is not dual of various entanglement measures. Furthermore, we show that the EWCS can be strictly larger than various entanglement measures at the leading order $O\left(N^{2}\right)$. It is particularly shown in a holographic configuration near to the saturation of the ArakiLieb inequality $[49,50]$. We also point out that the EWCS is also larger than another type of quantum correlation, the quantum discord [51,52]. It implies that the EWCS captures 
more than quantum entanglement in the entanglement wedge, and it must be sensitive to classical correlations as well.

Next, we introduce a series of holographic duals for the optimized correlation measures, which are akin to the EOP. This class includes two entanglement measures, the squashed entanglement [53] and the conditional entanglement of mutual information (CEMI) [54], and three total correlation measures, the EOP, the $Q$-correlation, and the $R$-correlation [55]. We show that the CEMI reduces to half of the holographic mutual information as the $R$-correlation does to the EWCS, when they are optimized over the geometrical extensions. These two duals thus do not lead to new geometrical object in the bulk.

However, we find that the holographic dual of the $Q$ correlation provides us with a new bulk measure of correlation inside the entanglement wedge, which we call the entanglement wedge mutual information (EWMI). This quantity appropriately satisfies all of the properties of the $Q$-correlation, as well as the strong superadditivity like the EWCS. Furthermore, we show that the EWMI can also strictly become larger than the various quantum correlation measures in the same holographic configurations. It again implies that classical correlations are included in holographic correlations and they are geometrically encoded in the entanglement wedge.

This paper is organized as follows: In Sec. II, we review the basic notion of the EWCS and information-theoretic correlation measures. In Sec. III, we show that the EWCS is strictly larger than various measures of quantum correlation in a holographic configuration near to the saturation of the Araki-Lieb inequality. In Sec. IV, we argue holographic duals of the optimized correlation measures, introduce the EWMI, and discuss the aspects of the EWMI. In Sec. V, we discuss some future problems. In the Appendix, we prove new inequalities of the multipartite EOP and the multipartite EWCS, complementing the work of [22].

\section{PRELIMINARIES}

\section{A. Entanglement wedge cross section}

In the present paper we deal with static spacetime for simplicity (a generalization to nonstatic spacetime is straightforward using the HRT formula [11,12] instead of the RT formula). The boundary subsystems are denoted by $A$ and $B$ and the entanglement wedge of $A B \equiv A \cup B$ (on a canonical time slice) is denoted by $\mathcal{M}_{A B}$ [16-18]. Given an entanglement wedge $\mathcal{M}_{A B}$, we may define the minimal cross section as follows $[14,15]$.

Suppose the boundary of $\mathcal{M}_{A B}$ is divided into two "subsystems" $\mathcal{A}$ and $\mathcal{B}$, i.e., $\partial \mathcal{M}_{A B}=\mathcal{A} \cup \mathcal{B}$ under the condition $\mathcal{A}=A \cup A^{\prime}, \quad \mathcal{B}=B \cup B^{\prime}$. We include the asymptotic AdS boundary and (if it exists) black hole horizon in the boundary of $\mathcal{M}_{A B}$. The EWCS of $\mathcal{M}_{A B}$, $E_{W}(A: B)$, is defined as the minimum of the holographic
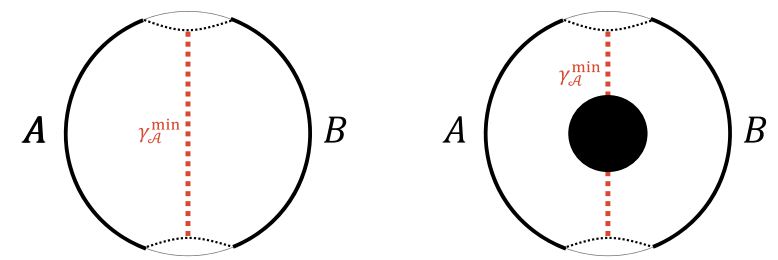

FIG. 1. The EWCS (red dashed lines) on a time slice of the entanglement wedge.

entanglement entropy $S_{\mathcal{A}}$ optimized over all possible partitions (Fig. 1)

$$
\begin{aligned}
E_{W}(A: B) & :=\min _{\mathcal{A}: \partial \mathcal{M}_{A B}=\mathcal{A} \cup \mathcal{B}, A \subset \mathcal{A}, B \subset \mathcal{B}} S_{\mathcal{A}} \\
& =\min _{\gamma_{\mathcal{A}}} \frac{\operatorname{Area}\left(\gamma_{\mathcal{A}}\right)}{4 G_{N}},
\end{aligned}
$$

where $\gamma_{\mathcal{A}}$ is the RT surface of $\mathcal{A}$. It gives a generalization of (1) for mixed states in the sense that $\gamma_{\mathcal{A}}$ reduces to the usual RT surface when $\rho_{A B}$ is a pure state. The EWCS always satisfies the inequalities $\frac{1}{2} I(A: B) \leq E_{W}(A: B) \leq \min \left\{S_{A}, S_{B}\right\}$, where $I(A: B):=S_{A}+S_{B}-S_{A B}$ is the mutual information. The above definition can be generalized to $n$-partite subsystems [22]. Remarkably, the EWCS can be regarded as a generalization of the area of a wormhole horizon in the canonical purification [46].

\section{B. Information-theoretic correlation measures}

The EWCS was originally conjectured to be dual to the EOP at the leading order $O\left(N^{2}\right)$. The EOP is defined for a bipartite state $\rho_{A B}$ by [19]

$$
E_{P}(A: B):=\min _{|\psi\rangle_{A A^{\prime} B B^{\prime}}} S_{A A^{\prime}}=\frac{1}{2} \min _{|\psi\rangle_{A A^{\prime} B B^{\prime}}} I\left(A A^{\prime}: B B^{\prime}\right),
$$

where the minimization is performed over all possible purifications. The information-theoretic properties of EOP $[19,56]$ are proven for the EWCS geometrically, including the multipartite cases [22]. Moreover, the surface/state correspondence of the tensor network description [21] allows us to find a heuristic derivation of $E_{W}=E_{P}$ [14].

The EOP, mutual information, $Q$-correlation, and $R$ correlation [55] (which are defined in Sec. IV) are monotonically nonincreasing under local operations (LO), but may increase by classical communication (CC). We call such non-negative quantities on $\rho_{A B}$ (bipartite) total correlation measures. On the other hand, entanglement measures are defined by monotonicity under local operations and classical communication (LOCC). There is a class of entanglement measures that satisfies additional axioms such as asymptotic continuity, which we collectively call (bipartite) axiomatic entanglement measures (see, e.g., [48]). There are various choices of additional 


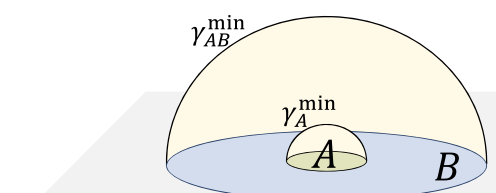

FIG. 2. A holographic configuration for which the Araki-Lieb inequality is saturated, $S_{A}+S_{A B}=S_{B}$.

axioms one can impose. In what follows we make a somewhat minimal requirement motivated by the uniqueness theorem [13]: They coincide with EE for pure states. This may be regarded as a normalization condition for different measures. Such a class includes, for instance, the distillable entanglement $E_{D}[57,58]$, the squashed entanglement $E_{s q}[53,59]$, the conditional entanglement of mutual information $E_{I}$ [54], the relative entropy of entanglement $E_{R E}$ [60], the entanglement cost $E_{C}$ [57,61], and the entanglement of formation $E_{F}$ [57]. There is another measure of quantum correlation, called the quantum discord $D$ [51,52]. It captures wider types of quantum correlation than quantum entanglement, and coincides with EE for pure states.

\section{EWCS IS NOT DUAL OF AXIOMATIC ENTANGLEMENT MEASURES}

First of all, we can use the generic upper bounds $E_{D}$, $E_{s q}, E_{I}, E_{R E}, D \leq I$ to exclude $E_{D}, E_{s q}, E_{I}, E_{R E}$, and $D$ as a dual candidate of $E_{W}$, since $E_{W}(A: B)>I(A: B)$ can be observed near to the $O(1)$ phase transition of $I(A: B)$ [14]. It already gives us intuition that the entanglement (or quantum correlation) measures are usually less than the EWCS in holographic CFTs. In this way, however, we cannot exclude $E_{C}$ and $E_{F}$ since they may exceed $I(A: B)$ [they can be greater than $I(A: B) / 2$ [62] ]. In order to do that, we consider another particular holographic setup as follows.

\section{A. The EWCS in the Araki-Lieb transition}

One of outstanding characteristics of holographic CFTs is the fact that the Araki-Lieb inequality,

$$
S_{A}+S_{A B} \geq S_{B},
$$

can be saturated at the leading order $O\left(N^{2}\right)$ in some particular configurations $[49,50]$. It is typically realized by a subsystem $A$ completely surrounded by sufficiently large $B$ (Fig. 2). Though the following discussion is valid for the more generic setups, we focus on a configuration in Poincaré $\mathrm{AdS}_{3}$ with the metric

$$
d s^{2}=\frac{d z^{2}-d t^{2}+d x^{2}}{z^{2}} .
$$
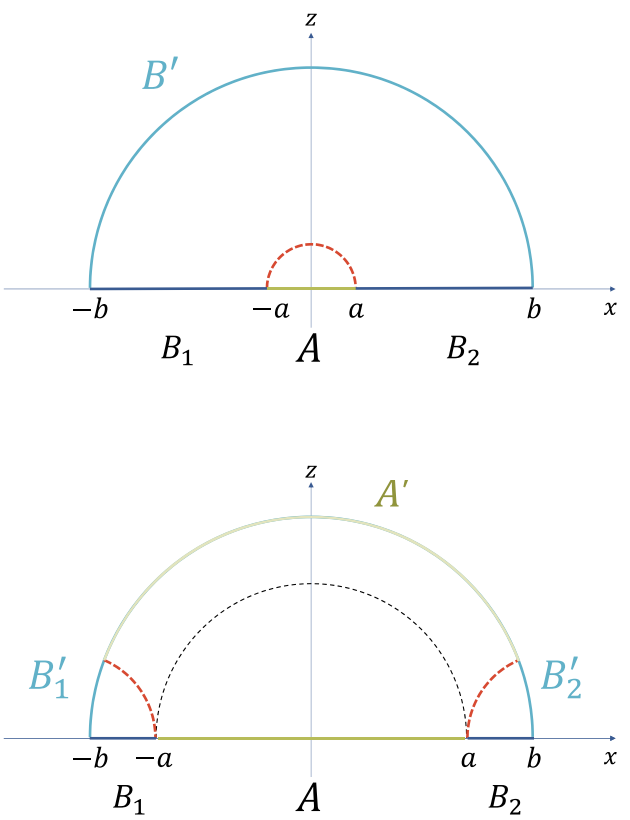

FIG. 3. The two configurations of the EWCS $E_{W}(A: B)=$ $E_{W}\left(A: B_{1} B_{2}\right)$, denoted by the orange dashed line, for the symmetric setup in the Poincaré $\mathrm{AdS}_{3}$. The left (right) configuration is preferred when the relative size $p<p_{\mathrm{EW}}^{*}\left(p>p_{\mathrm{EW}}^{*}\right)$. The primed symbols allocated on the upper semicircle denote the partition in (3) with $\mathcal{A}=A \cup A^{\prime}$ and $\mathcal{B}=B_{1} \cup B_{2} \cup B_{1}^{\prime} \cup B_{2}^{\prime}$.

Suppose the subsystems $A$ and $B$ are given by $A=$ $[-a, a], B=[-b,-a] \cup[a, b] \equiv B_{1} \cup B_{2}$ for $0<a<b$ w.l.o.g. We also define the relative size of subsystems by $p \equiv \frac{a}{b}$ for $p \in(0,1)$. The mutual information $I(A: B)$ exhibits a phase transition due to that of $S_{B}=S_{B_{1} B_{2}}$ depending on the relative size $p$. The connected phase $I\left(B_{1}: B_{2}\right)>0$ is preferred if $p$ is small, and the disconnected phase $I\left(B_{1}: B_{2}\right)=0$ is if it is large. Thus $I(A: B)$ can be computed as

$$
\begin{aligned}
I(A: B) & =S_{A}+S_{B}-S_{A B} \\
& =\min \left\{\frac{2 c}{3} \log \frac{2 a}{\epsilon}, \frac{2 c}{3} \log \frac{\sqrt{a / b}(b-a)}{\epsilon}\right\},
\end{aligned}
$$

where $c$ is the central charge of holographic two-dimensional CFTs and $\epsilon$ is the UV cutoff. It is divergent since we are taking the adjacent limit. The phase transition point of $I(A: B)$ can be read off as

$$
p_{\mathrm{MI}}^{*} \equiv \frac{a_{\mathrm{MI}}^{*}(b)}{b}=3-2 \sqrt{2} .
$$

The Araki-Lieb inequality is saturated for $0<p<p_{\mathrm{MI}}^{*}$ but not for $p_{\mathrm{MI}}^{*}<p<1$.

The EWCS also exhibits a phase transition depending on the relative size of $A$ (Fig. 3). The formula for the EWCS in Poincaré $\mathrm{AdS}_{3}$ is given in [14] by 


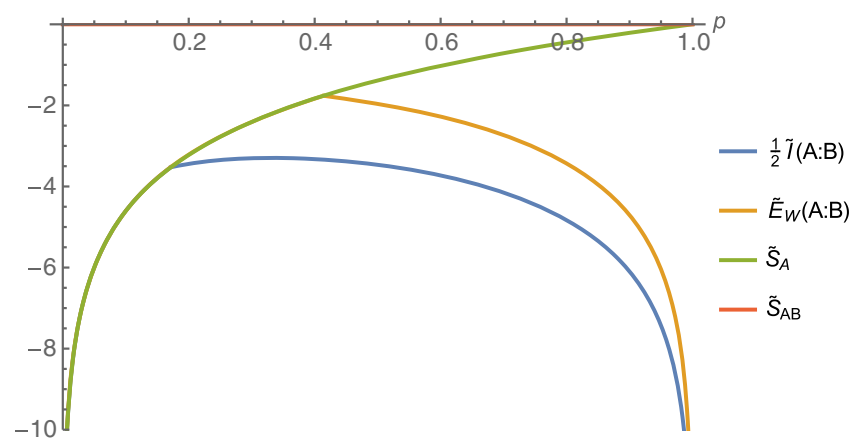

FIG. 4. Half of the mutual information and the EWCS for the Araki-Lieb transition (normalized by subtracting $S_{A B}$ ).

$$
\begin{aligned}
E_{W} & =\min \left\{S_{A}, 2 E_{W}\left(A B_{1}: B_{2}\right)\right\} \\
& =\min \left\{\frac{c}{3} \log \frac{2 a}{\epsilon}, \frac{c}{3} \log \left[\frac{b^{2}-a^{2}}{b \epsilon}\right]\right\} .
\end{aligned}
$$

The phase transition of EWCS therefore happens at

$$
p_{\mathrm{EW}}^{*} \equiv \frac{a_{\mathrm{EW}}^{*}(b)}{b}=\sqrt{2}-1 \text {. }
$$

The phase transition points of the mutual information and the EWCS do not match, and the strict inequality $p_{\mathrm{MI}}^{*}<p_{\mathrm{EW}}^{*}$ holds (Fig. 4). This means that the EWCS saturates its upper bound $E_{W}(A: B)=S_{A}$ while the ArakiLieb inequality is not saturated for $p \in\left(p_{\mathrm{MI}}^{*}, p_{\mathrm{EW}}^{*}\right)$. This observation provides us a crucial benchmark: A correlation measure $E$ cannot be dual to the EWCS if $E(A: B)=S_{A}$ automatically implies the saturation of the Araki-Lieb inequality $S_{A}+S_{A B}=S_{B}$.

The Araki-Lieb inequality is also holographically saturated in the global Banados-Teitelboim-Zanelli (BTZ) black hole

$$
\begin{gathered}
d s^{2}=\frac{f^{-1}(z) d z^{2}-f(z) d t^{2}+d x^{2}}{z^{2}}, \\
f(z)=1-\frac{z^{2}}{z_{H}^{2}},
\end{gathered}
$$

with the inverse temperature $\beta=2 \pi z_{H}$ and the periodic boundary condition $x \simeq x+2 \pi$. We choose $A=[-l / 2, l / 2]$ for $l \in(0, \pi)$ and $B$ as the remainder. It exhibits the ArakiLieb saturation when the size of $A$ is small enough. We find the phase transition points (see, e.g., $[14,15,49]$ )

$$
\begin{gathered}
l_{\mathrm{MI}}^{*}\left(z_{H}\right)=\pi-z_{H} \log \cosh \left(\frac{\pi}{z_{H}}\right), \\
l_{\mathrm{EW}}^{*}\left(z_{H}\right)=2 z_{H} \log (1+\sqrt{2}) .
\end{gathered}
$$

This leads to $l_{\mathrm{MI}}^{*}\left(z_{H}\right)<I_{\mathrm{EW}}^{*}\left(z_{H}\right)$ for any $z_{H}>0$, which confirms the above conclusion.

\section{B. The axiomatic entanglement measures and the Araki-Lieb saturation}

We can now cite the following fact: the entanglement of formation saturates its upper bound $E_{F}(A: B) \leq S_{A}$ if and only if the Araki-Lieb inequality is saturated [63]. This immediately means that $E_{F}$ is not dual of $E_{W}$ from the above observation. Furthermore, this statement can be generalized to another measure $E$, which satisfies (i) the monotonicity $E\left(A: B_{1} B_{2}\right) \geq E\left(A: B_{1}\right)$, (ii) $E(A: B)=S_{A}=S_{B}$ for pure states, and (iii) $E \leq E_{F}$. Indeed, the saturation $E(A: B)=S_{A}$ leads to $E_{F}(A: B)=S_{A}$ from (iii), which is equivalent to the Araki-Lieb saturation. The opposite is shown by using the unique structure (up to isometries) of states that saturate the Araki-Lieb inequality [64]

$$
\rho_{A B}=|\psi\rangle\left\langle\left.\psi\right|_{A B_{L}} \otimes \rho_{B_{R}},\right.
$$

where the Hilbert space of $B$ is decomposed into $\mathcal{H}_{B}=$ $\mathcal{H}_{B_{L}} \otimes \mathcal{H}_{B_{R}}$. This form with (i) and (ii) leads to the saturation by $S_{A} \geq E\left(A: B_{L} B_{R}\right) \geq E\left(A: B_{L}\right)=S_{A}$. This class of correlation measures especially includes the entanglement $\operatorname{cost} E_{C}$, and the other entanglement measures mentioned at the beginning of this section as well. Thus we can also exclude $E_{C}$ and the other measures as a dual candidate of $E_{W}$.

In addition, it also means that this class of entanglement measures must be strictly less than $E_{W}$ for the states of $p \in\left(p_{\mathrm{MI}}^{*}, p_{\mathrm{EW}}^{*}\right)$. Since there seems to be no reason to believe that these states are singular among the holographic states, we argue that the EWCS is generically larger than these entanglement measures in holographic CFTs (unless the Araki-Lieb inequality is saturated).

\section{Interpretation from the holographic entanglement of purification}

By contrast, the EOP evades the above criteria since (iii) does not hold, and still deserves consideration as a possible dual of the EWCS. In addition, there exists a class of states for which $E_{P}(A: B)=S_{A}$, but the Araki-Lieb inequality is not saturated [65]. Similarly, the logarithmic negativity, the odd entropy, and the reflected entropy do not satisfy (iii), and we expect that they also coincide with $S_{A}$ with an appropriate coefficient for some mixed states without the Araki-Lieb saturation.

Furthermore, we can understand the behavior of the EWCS through the Araki-Lieb transition based on the surface/state correspondence. First, we note a remarkable equality that holds after the phase transition $p>p_{\mathrm{EW}}^{*}$,

$$
E_{W}\left(A: B_{1} B_{2}\right)=E_{W}\left(A B_{1}: B_{2}\right)+E_{W}\left(A B_{2}: B_{1}\right) .
$$

This equation can be explained as follows: the two configurations $p>p_{\mathrm{EW}}^{*}$ and $p<p_{\mathrm{EW}}^{*}$ are equivalent to whether the correlation $I\left(\mathcal{B}_{1}: \mathcal{B}_{2}\right)$ vanishes or not. For $p>p_{\mathrm{EW}}^{*}$, we 
see $I\left(\mathcal{B}_{1}: \mathcal{B}_{2}\right)=0$, and it immediately leads to the unique form (up to isometries on $\mathcal{H}_{\mathcal{A}}$ ) of any purification [66],

$$
|\psi\rangle_{\mathcal{A B}_{1} \mathcal{B}_{2}}=\left|\phi^{1}\right\rangle_{\mathcal{A B}} \otimes\left|\phi^{2}\right\rangle_{\mathcal{A B}_{2}}
$$

This form of optimal purification, common to each of the three EWCSs, clearly establishes the equality (16).

On the other hand, if $p<p_{\mathrm{EW}}^{*}$, remaining correlation $I\left(\mathcal{B}_{1}: \mathcal{B}_{2}\right)>0$ drastically changes the structure of purifications from (17). In this case, the optimal purification would be simply given by the standard purification [19], i.e., setting $A^{\prime}$ as empty. In this sense, the phase transition point $p_{\mathrm{EW}}^{*}$ is thus understood as a point at which the standard purification switches with the decoupled purification (17) as the optimal purification.

\section{HOLOGRAPHIC DUALS OF THE OPTIMIZED CORRELATION MEASURES}

We observed that the EWCS cannot be the dual of any axiomatic entanglement measures. Then a natural question is as follows: Is there any axiomatic entanglement measure that deserves a geometrical dual?

\section{A. Holographic dual of the optimized entanglement measures}

Here we discuss two possible candidates: the squashed entanglement $E_{s q}$ [53] and the conditional entanglement of mutual information $E_{I}$ [54]. Their definitions are reminiscent of the EOP (4). The squashed entanglement is defined as

$$
\begin{aligned}
E_{s q}(A: B) & :=\frac{1}{2} \min _{\rho_{A B E}} I(A: B \mid E) \\
& =\frac{1}{2} I(A: B)-\frac{1}{2} \max _{\rho_{A B E}} I_{3}(A, B, E),
\end{aligned}
$$

where $\rho_{A B E}$ is an extension such that $\operatorname{Tr}_{E} \rho_{A B E}=\rho_{A B}$, and $I_{3}(A, B, C)=S_{A}+S_{B}+S_{C}-S_{A B}-S_{B C}-S_{C A}+S_{A B C}$ is the tripartite information.

We now impose a crucial assumption to find a possible geometrical dual of $E_{s q}$ : Performing the minimization over a class of extensions that have classical geometrical duals is sufficient to achieve the minimum. It implies that the monogamy of mutual information $I_{3}(A, B, E) \leq 0$ [47] must hold for the extensions $\rho_{A B E}$. A holographic dual of the squashed entanglement is then given by half of the holographic mutual information [47],

$$
E_{s q}(A: B)=\frac{1}{2} I(A: B) .
$$

This is achieved by a trivial extension $E=\varnothing$. This relation implies that the holographic mutual information should satisfy the properties of the squashed entanglement, such as the monogamy relation $E_{s q}(A: B C) \geq E_{s q}(A: B)+E_{s q}(A: C)$ [67], which is generically considered as a characteristic of quantum entanglement. The holographic mutual information indeed satisfies the monogamy relation as mentioned above. It is worth noting that the saturation of $E_{s q} \leq \frac{1}{2} I$ occurs if $\rho_{A B}$ saturates Araki-Lieb inequality, but this is not the only possibility [65].

The relation (20), or the monogamy property of the mutual information, suggests a striking conclusion: the mutual information captures only quantum entanglement in holography, even though it is usually a total correlation measure [47].

We give support for this argument by elaborating on the conditional entanglement of mutual information $E_{I}$ [54]. It is defined by

$$
\begin{aligned}
E_{I}(A: B):= & \frac{1}{2} \min _{A B A^{\prime} B^{\prime}}\left(I\left(A A^{\prime}: B B^{\prime}\right)-I\left(A^{\prime}: B^{\prime}\right)\right) \\
= & \frac{1}{2} I(A: B)+\frac{1}{2} \min _{A B A^{\prime} B^{\prime}}\left(I\left(A A^{\prime}: B B^{\prime}\right)\right. \\
& \left.-I(A: B)-I\left(A^{\prime}: B^{\prime}\right)\right),
\end{aligned}
$$

where $\rho_{A B A^{\prime} B^{\prime}}$ is again any extension of $\rho_{A B}$. It is an additive measure of quantum entanglement [54]. Suppose the monogamy of mutual information for some geometric extensions $\rho_{A A^{\prime} B B^{\prime}}$ is enough to find the minimum. Then we find $I\left(A A^{\prime}: B B^{\prime}\right)-I(A: B)-I\left(A^{\prime}: B^{\prime}\right) \geq I\left(A: B^{\prime}\right)+$ $I\left(B: A^{\prime}\right) \geq 0$, which leads to the holographic dual of the CEMI as half of the holographic mutual information (with a trivial extension $A^{\prime} B^{\prime}=\varnothing$ ),

$$
E_{I}(A: B)=\frac{1}{2} I(A: B)
$$

It again implies that the holographic mutual information only captures quantum entanglement. This is in contrast to the EWCS, which still captures classical correlations in holography. Indeed, it was pointed out in [32] that the EOP could be more sensitive to classical correlations than the mutual information.

These proposals about $E_{s q}$ and $E_{I}$ are obviously consistent with the Araki-Lieb transition discussed above, since the holographic dual of $E_{s q}$ and $E_{I}$ would be the holographic mutual information itself.

We emphasize the fact that two differently defined measures of entanglement reduce to the same quantity $\frac{1}{2} I$ in holography. To our knowledge, there seems to be no obstruction to speculate that the other entanglement measures such as $E_{C}$ and $E_{F}$ also coincide with $\frac{1}{2} I$. We leave investigating their holographic duals as an interesting future work. 


\section{B. Holographic duals of the optimized total correlation measures}

All of the correlation measures $E_{P}, E_{s q}, E_{I}$ are defined as the minimum of a linear combination of von Neumann entropies over all possible purifications or extensions. This class of correlation measures is called the optimized correlation measures [55]. There are two other such measures, the $Q$-correlation and the $R$-correlation, introduced in [55]

$$
\begin{aligned}
E_{Q}(A: B) & :=\frac{1}{2} \min _{\rho_{A B E}}\left(S_{A}+S_{B}+S_{A E}-S_{B E}\right) \\
& \equiv \min _{\rho_{A B E}} f^{Q}(A, B, E) . \\
E_{R}(A: B):= & \frac{1}{2} \min _{\rho_{A B E}}\left(S_{A B}+2 S_{A E}-S_{A B E}-S_{E}\right) \\
& \equiv \min _{\rho_{A B E}} f^{R}(A, B, E) .
\end{aligned}
$$

The symmetry between $A$ and $B$ becomes obvious in the equivalent expression in terms of purifications $\left(E \equiv A^{\prime}\right)$,

$$
E_{Q}(A: B)=\frac{1}{2} \min _{|\psi\rangle_{A A^{\prime} B B^{\prime}}}\left(S_{A}+S_{B}+\frac{S_{A A^{\prime}}+S_{B B^{\prime}}-S_{B A^{\prime}}-S_{A B^{\prime}}}{2}\right)
$$

$$
\begin{aligned}
& \equiv \min _{|\psi\rangle_{A A^{\prime} B B^{\prime}}} f^{Q}\left(A, A^{\prime}, B, B^{\prime}\right) . \\
E_{R}(A: B) & =\frac{1}{2} \min _{|\psi\rangle_{A A^{\prime} B B^{\prime}}}\left(S_{A B}+S_{A A^{\prime}}+S_{B B^{\prime}}-S_{A^{\prime}}-S_{B^{\prime}}\right) \\
& \equiv \min _{|\psi\rangle_{A A^{\prime} B B^{\prime}}} f^{R}\left(A, A^{\prime}, B, B^{\prime}\right) .
\end{aligned}
$$

The $Q$-correlation and the $R$-correlation are nonincreasing under local operations, but not necessarily under LOCC. They satisfy the inequality [55]

$$
\frac{1}{2} I \leq E_{Q}, \quad E_{R} \leq E_{P} .
$$

We note a close relationship between the $R$-correlation and the CEMI, which is clear from the following expression of $E_{R}$,

$$
E_{R}(A: B)=\frac{1}{2} \min _{|\psi\rangle_{A A^{\prime} B B^{\prime}}}\left(I\left(A A^{\prime}: B B^{\prime}\right)-I\left(A^{\prime}: B^{\prime}\right)\right) .
$$

It is similar to the CEMI (21), though the minimization of the CEMI is performed over all possible extensions.

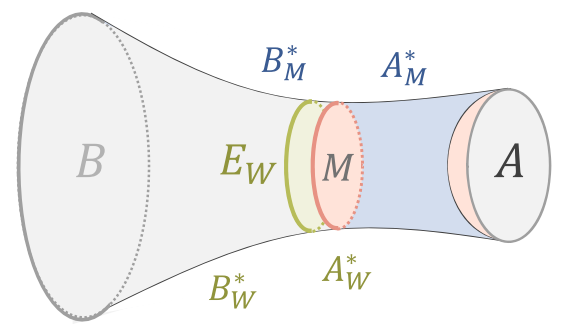

FIG. 5. The entanglement wedge mutual information $E_{M}$ in the entanglement wedge. In the above picture, $E_{M}$ is given by the area of red codimension-2 surfaces subtracted by the area of blue codimension-2 surface (divided by $2 \cdot 4 G_{N}$ ), which may be understood as the mutual information $\frac{1}{2} I(A: M)$. The symmetry $E_{M}(A: B)=E_{M}(B: A)$ stems from the fact that the RT surface of $S_{B A^{\prime}}$ and $S_{A B^{\prime}}$ has the same configurations. The optimal partition $A_{M}^{*}$ and $B_{M}^{*}$ of the EWMI located on the RT surface of $S_{A B}$ is not necessarily equivalent to these $A_{W}^{*}$ and $B_{W}^{*}$ of the EWCS.

\section{The holographic counterparts}

Here we investigate holographic duals of the $Q$ correlation and the $R$-correlation. The definition of the holographic dual candidate of $E_{Q}$ is stated as follows (we focus on static geometries):

Given an entanglement wedge $\mathcal{M}_{A B}$, divide its boundary into $\partial \mathcal{M}_{A B}=\mathcal{A} \cup \mathcal{B}$ so that $\mathcal{A}=A \cup A^{\prime}$ and $\mathcal{B}=B \cup B^{\prime}$. Then minimize the combination of holographic entanglement entropy $f^{Q}\left(A, A^{\prime}, B, B^{\prime}\right)$ over all possible partitions. We define the minimum as the EWMI, denoted by $E_{M}$,

$$
E_{M}(A: B):=\min _{A^{\prime} \cup B^{\prime}} f^{Q}\left(A, A^{\prime}, B, B^{\prime}\right) .
$$

An example of the EWMI is depicted in Fig. 5. It may be regarded as the half of the mutual information between $A$ (or $B$ ) and the subsystem $M$ assigned to the codimention-2 cross section of $S_{A A^{\prime}}\left(=S_{B B^{\prime}}\right)$.

Generically, the EWMI requires us to consider many complicated configurations of $A^{\prime}$ and $B^{\prime}$ in order to minimize $f^{Q}$. For some simple cases, however, such as the two disjoint intervals in $\mathrm{AdS}_{3} / \mathrm{CFT}_{2}$ or the (symmetric) Araki-Lieb saturating configurations, there is an intuitive way to compute $E_{M}$ owing to the symmetry of setup: Minimize (half of) the mutual information $\max \{I(A: M), I(B: M)\}$ over all possible choices of the cross sections,

$$
E_{M}(A: B)=\frac{1}{2} \min _{M}(\max \{I(A: M), I(B: M)\}),
$$

where $M$ corresponds to the cross section of some partition $A^{\prime} \cup B^{\prime}$. This form also clarifies a useful relation

$$
I\left(A: M^{*}\right)=I\left(B: M^{*}\right),
$$

for at least one of the optimal cross sections $M^{*}$. Note that the optimal purification for $E_{M}$ is not necessarily unique; nor does it necessarily agree with that of $E_{W}$ (Fig. 5). We see both 
concrete examples in the below discussion of the Araki-Lieb transition of $E_{M}$. There is another suggestive form of $E_{M}$ for these cases,

$$
\begin{aligned}
& E_{M}(A: B) \\
& =\frac{1}{2}\left[\frac{1}{2} I(A: B)+\min _{A^{\prime} \cup B^{\prime}}\left(S_{A A^{\prime}}+\frac{I\left(A: B^{\prime}\right)+I\left(B: A^{\prime}\right)}{2}\right)\right],
\end{aligned}
$$

where we have used $I\left(A^{\prime}: B^{\prime}\right)=0$, which holds for the ancillary subsystems on the RT surface. At least one of $I\left(A: B^{\prime}\right)$ and $I\left(B: A^{\prime}\right)$ must vanish at this point because the whole system is homologically trivial. Moreover, the balancing condition (36) is equivalent to the condition $I\left(A: B^{*}\right)=I\left(B: A^{*}\right)$. Thus we can conclude that both $I\left(A: B^{\prime}\right)$ and $I\left(B: A^{\prime}\right)$ should vanish for the balanced optimal partition. As a result, we reach a formula

$$
E_{M}(A: B)=\frac{1}{2}\left[\frac{1}{2} I(A: B)+S_{A A_{b}^{*}}\right],
$$

where $A_{b}^{*}$ is the balanced optimal partition. We may define the deviation from the EWCS due to the balancing term $S_{B A^{\prime}}$ as

$$
D_{b}(A: B):=S_{A A_{b}^{*}}-E_{W}(A: B) \geq 0 .
$$

We check this formula (38) by direct computation in the Araki-Lieb transition.

A caveat is that neither formula (35) nor (38) is necessarily valid for any configurations, and there possibly exists other types of optimal configurations of $A^{\prime}$ and $B^{\prime}$ for more complicated subsystems. Indeed, for example, if we set $\left|B_{1}\right|>\left|B_{2}\right|$ in the Araki-Lieb saturating configuration, $E_{M}$ can be realized by an optimal configuration neither of $I\left(A: M^{*}\right)$ nor $I\left(B: M^{*}\right)$, but of a combination $I\left(A: M_{A}^{*}\right)+I\left(B: M_{B}^{*}\right)$ where $M_{A}^{*} \cup M_{B}^{*}=M$. Such a configuration is not preferred for the disjoint two intervals [68] or for the symmetric Araki-Lieb configuration. This example indicates that we need to replace $\max \{I(A: M), I(B: M)\}$ in (35) with $\max _{M_{A} \cup M_{B}=M}\left\{I\left(A: M_{A}\right)+I\left(B: M_{B}\right)\right\}$ in general. We leave proving or disproving it for generic configurations as an important future work.

The EWMI satisfies the properties of $E_{Q}$. For example, it cannot be greater than the EWCS,

$$
E_{M} \leq E_{W}
$$

which must hold to be consistent with $E_{W}=E_{P}$ from (32). One can prove this inequality by drawing a picture, but an easier way is to use the von Neumann entropy to represent the corresponding geometrical areas. Suppose the optimal partition of $E_{W}$ is given by $A_{W}^{*}$ and $B_{W}^{*}$. Then we can show $E_{W}=S_{A A_{W}^{*}} \geq \frac{1}{2}\left(S_{A}+S_{B}+S_{A A_{W}^{*}}-S_{B A_{W}^{*}}\right) \geq E_{M}$, where we have used strong subadditivity.

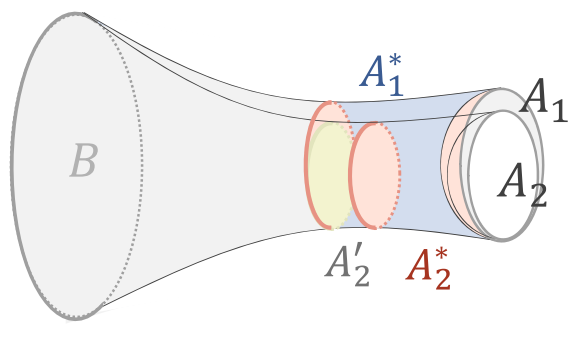

FIG. 6. The extensivity $E_{M}\left(A_{1}: B\right) \geq E_{M}\left(A_{2}: B\right)$ for $A_{1} \supset A_{2}$. We abbreviate the $B^{\prime}$ labels. From the optimal partition $A_{1}^{*}$ for $A_{1}$, one can induce a partition $A_{2}^{\prime}$ on $\partial \mathcal{M}_{A_{2} B}$ so that $A_{1}^{*} \cap \gamma_{A_{2} B}=A_{2}^{\prime} \cap \gamma_{A_{2} B}$. Then $E_{M}\left(A_{1}: B\right) \geq f^{Q}\left(A_{2}, B, A_{2}^{\prime}\right)$ holds due to the minimality of the RT surface, and $f^{Q}\left(A_{2}, B, A_{2}^{\prime}\right) \geq$ $E_{M}\left(A_{2}: B\right)$ is clear by definition.

Similarly, $E_{M}$ cannot be less than half of the holographic mutual information,

$$
\frac{1}{2} I \leq E_{M}
$$

It is clear from (38) as $S_{A A_{b}^{*}} \geq E_{W}(A: B) \geq \frac{1}{2} I(A: B)$. These properties also guarantee that $E_{M}(A: B)=S_{A}=S_{B}$ for pure states, and that $E_{M}$ vanishes if and only if $I(A: B)=0$ (with $A^{*}=\gamma_{A}$ and $B^{*}=\gamma_{B}$ ). It also shows the extensivity $E_{M}\left(A_{1}: B\right) \geq E_{M}\left(A_{2}: B\right)$ when $A_{1} \supset A_{2}$ (Fig. 6). The additivity $E_{M}\left(\rho_{A_{1} B_{1}} \otimes \sigma_{A_{2} B_{2}}\right)=E_{M}\left(\rho_{A_{1} B_{1}}\right)+E_{M}\left(\sigma_{A_{2} B_{2}}\right)$ is also clear because the decoupled state corresponds to disjoint geometries. All of these consistent properties tempt us to propose the relation [at the leading order $O\left(N^{2}\right)$ ]

$$
E_{Q}=E_{M}
$$

In pure $\mathrm{AdS}_{3}$, the $E_{M}$ for two disjoint intervals has a simple expression (Fig. 7). In such cases, the optimal partition coincides with that of $E_{W}$, as it is obvious from the conformal symmetry. Thus $E_{M}$ becomes just the average of $\frac{1}{2} I$ and $E_{W}$ by (38),

$$
E_{M}(A: B)=\frac{1}{2}\left[\frac{1}{2} I(A: B)+E_{W}(A: B)\right] .
$$

From this expression, we can easily confirm all of the properties of $E_{M}$ mentioned above. This expression is not
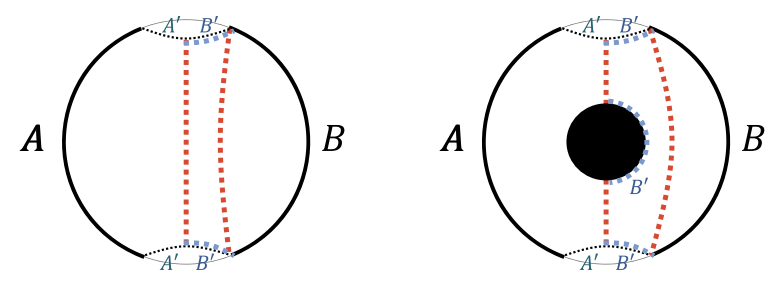

FIG. 7. The $E_{M}$ in the pure global $\mathrm{AdS}_{3}$ (left) and in the global BTZ (right) for the symmetric two disjoint intervals, in which $E_{M}=\frac{1}{2}\left(\frac{1}{2} I+E_{W}\right)$. In the vacuum, one can map the two disjoint subsystems into this setup by the conformal symmetry. 
necessarily true in generic setups such as three or more multipartite intervals or black hole geometry.

Surprisingly, the EWMI also satisfies the strong superadditivity

$$
E_{M}\left(\rho_{A_{1} A_{2} B_{1} B_{2}}\right) \geq E_{M}\left(\rho_{A_{1} B_{1}}\right)+E_{M}\left(\rho_{A_{2} B_{2}}\right),
$$

which can be proven geometrically (Fig. 8). It is similar to the proof of the strong superadditivity of $E_{W}$ [14]. The relation (44) is not a generic property of $E_{Q}$. Thus we may regard it as a characteristic of holographic correlations, as with the holographic entropy cone [47,69-72].

The dual of $E_{R}$ is defined in the same manner, replacing $f^{Q}$ with $f^{R}$ in the above procedure. However, it turns out that this definition is equivalent to that of the EWCS. It stems from the fact we implicitly used in the definition of $E_{M}$ (and $E_{W}$ ) that it is sufficient to consider the ancillary systems $A^{\prime}$ and $B^{\prime}$ located only on the RT surface $S_{A B}$ for minimization. For such subsystems we find $I\left(A^{\prime}: B^{\prime}\right)=S_{A^{\prime}}+S_{B^{\prime}}-S_{A B}=0$, resulting in $E_{R}=E_{P}=$ $E_{W}$ from (33). We also state it as a holographic proposal

$$
E_{R}=E_{W}
$$

The additivity of the EWCS is consistent with that of the $R$ correlation [55]. The relation between $E_{R}$ and $E_{I}$ then gives an interesting perspective on the geometrical extensions: if only pure geometries are available, the correlations can reduce to $E_{W}$ at most. If mixed geometries are also allowed, then the inaction gives a further reduction to $\frac{1}{2} I$.

\section{The EWMI in the Araki-Lieb transition}

Let us study $E_{M}$ in the Araki-Lieb transition discussed in Sec. III A in detail. First, we remark that $E_{M}=S_{A}$ should
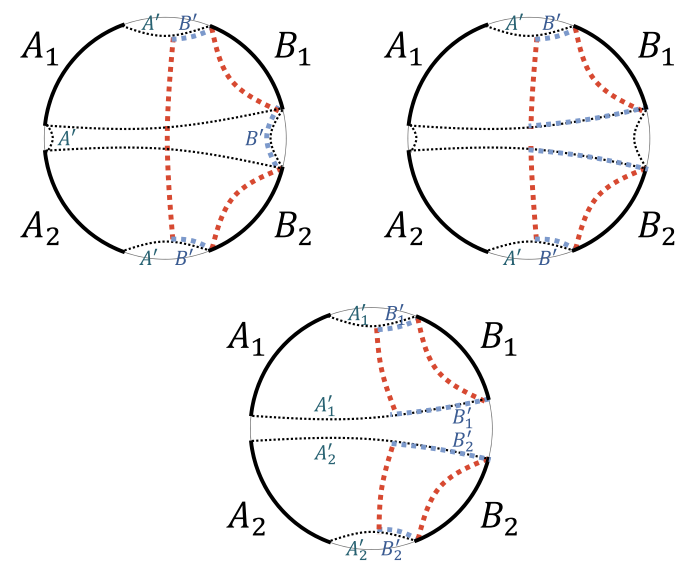

FIG. 8. A proof of the strong superadditivity for $E_{M}$. The left figure corresponds to $E_{M}\left(\rho_{A_{1} A_{2} B_{1} B_{2}}\right)$, and the right one corresponds to $E_{M}\left(\rho_{A_{1} B_{1}}\right)+E_{M}\left(\rho_{A_{2} B_{2}}\right)$. As the total of the areas, (left) $\geq$ (middle) $\geq$ (right) is obvious (plus sign for red and minus sign for blue). hold for $p<p_{\mathrm{MI}}^{*}$ from the inequality $\frac{1}{2} I \leq E_{M} \leq E_{W}$, while it also can be checked by direct computation. For $p>p_{\mathrm{MI}}^{*}$, the situation is more complicated than $E_{W}$ due to the four configurations of $S_{A A^{\prime}}-S_{B A^{\prime}}$. For simplicity, we fix the size $b$ to unit size in the setup and always deal with the relative size $p$ as the parameter.

The two phases of $S_{A A^{\prime}}$ and the two phases of $S_{B A^{\prime}}$ are depicted in Fig. 9. The minimal configuration depends not only on the parameter $p$ but also on the size of $A^{\prime}$, parametrized by $q \in(0,1)$. We can easily find out the minimal configurations in the extremal cases: in the small $A^{\prime}$ limit $(q \rightarrow 0)$, the phase (A1) for $S_{A A^{\prime}}$ and the phase (B1) for $S_{B A^{\prime}}$ are preferred [recall $I\left(B_{1}: B_{2}\right)=0$ for $p>p_{\mathrm{MI}}^{*}$ ]. Similarly, we have the phase (A2) for $S_{A A^{\prime}}$ and the phase (B2) for $S_{B A^{\prime}}$ in the large $A^{\prime}$ limit $(q \rightarrow 1)$. Therefore, as we increase $q$ from 0 to 1 , we see phase transitions of $S_{A A^{\prime}}-$ $S_{B A^{\prime}}$ for the fixed $p>p_{\mathrm{MI}}^{*}$ in either path,

$$
\begin{aligned}
& (\mathrm{I})(\mathrm{A} 1, \mathrm{~B} 1) \rightarrow(\mathrm{A} 1, \mathrm{~B} 2) \rightarrow(\mathrm{A} 2, \mathrm{~B} 2), \\
& (\mathrm{II})(\mathrm{A} 1, \mathrm{~B} 1) \rightarrow(\mathrm{A} 2, \mathrm{~B} 1) \rightarrow(\mathrm{A} 2, \mathrm{~B} 2) .
\end{aligned}
$$

Note that $S_{B A^{\prime}}$ is always in (B2) regardless of $q$ for $p<p_{\mathrm{MI}}^{*}$.

It is not hard to show that increasing $q$ may decrease $S_{A A^{\prime}}-S_{B A^{\prime}}$ only in the phase (A2, B1). In the phase (A1, B1), changing $q$ has no effect at all, and in the phase (A1, B2) and (A2, B2), increasing $q$ does increase $S_{A A^{\prime}}-S_{B A^{\prime}}$. Therefore, a nontrivial optimal partition for $E_{M}$ is observed only when the phase transition follows the path (II).

With this in mind, we find the phase transition points $q^{*}$ of $S_{A A^{\prime}}$ and $S_{B A^{\prime}}$ as a function of $p$,

$$
q_{A A^{\prime}}^{*}(p)=\frac{(1-p)^{2}}{4 p},
$$
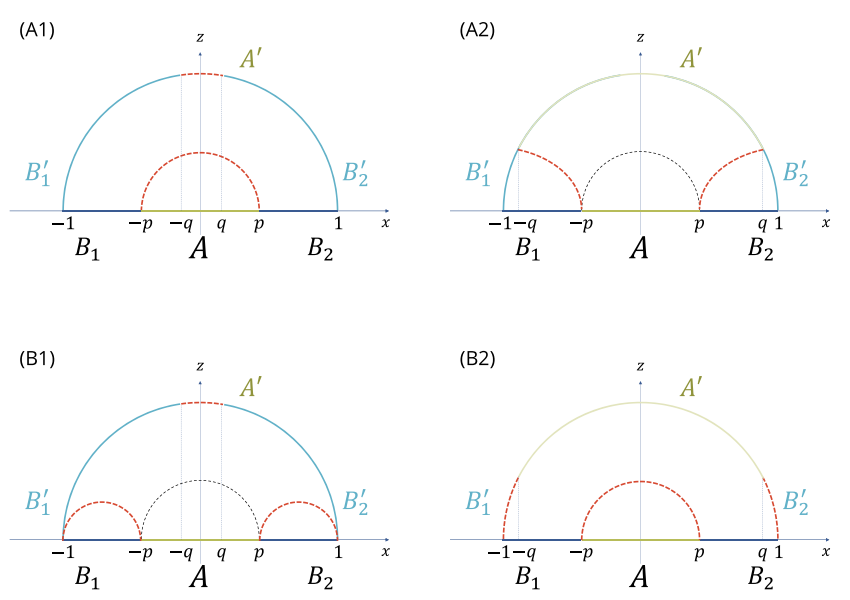

FIG. 9. The two phases of $S_{A A^{\prime}}$ (top panels) and these of $S_{B A^{\prime}}$ (bottom panels). The RT surfaces of $S_{A A^{\prime}}$ and $S_{B A^{\prime}}$ are denoted as red dashed lines. 


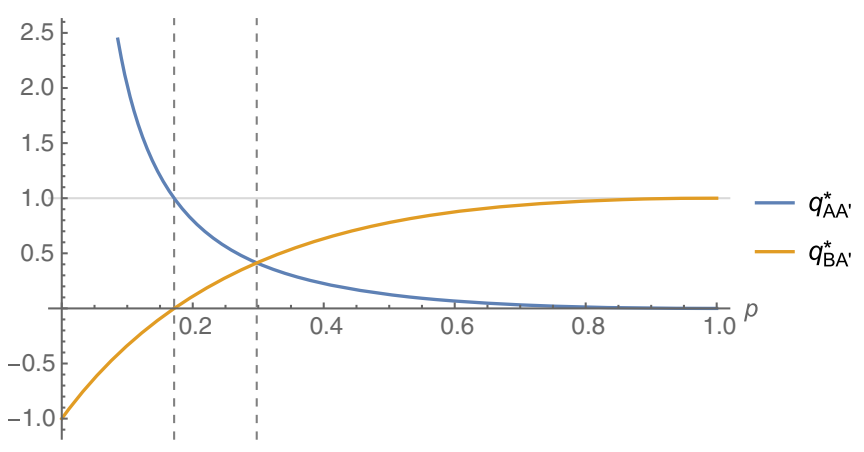

FIG. 10. The phase transition points of $S_{A A^{\prime}}$ (blue) and $S_{B A^{\prime}}$ (yellow) with respect to the size $q$. The vertical dashed lines denote $p_{\mathrm{MI}}^{*}=3-2 \sqrt{2} \simeq 0.17$ and $p_{\mathrm{EM}}^{*}=-1+2 \sqrt{2}-$ $2 \sqrt{2-\sqrt{2}} \simeq 0.30$.

$$
q_{B A^{\prime}}^{*}(p)=-\frac{1-6 p+p^{2}}{(1+p)^{2}}
$$

These are plotted in Fig. 10. The region $q<q_{A A^{\prime}}^{*}$ corresponds to the phase (A1) for $S_{A A^{\prime}}$, and the region $q<q_{B A^{\prime}}^{*}$ to the phase (B1) for $S_{B A^{\prime}}$. The phase transition of $E_{M}$ happens at the crossing point $p_{\mathrm{EM}}^{*}$ at which $q_{A A^{\prime}}^{*}\left(p_{\mathrm{EM}}^{*}\right)=$ $q_{B A^{\prime}}^{*}\left(p_{\mathrm{EM}}^{*}\right)$ holds,

$$
p_{\mathrm{EM}}^{*}=-1+2 \sqrt{2}-2 \sqrt{2-\sqrt{2}} \simeq 0.30
$$

The ancillary system $A^{\prime}$ of any size $q \leq q_{B A^{\prime}}^{*}$ achieves the minimum $E_{M}=S_{A}$ for $p<p_{\mathrm{EM}}^{*}$. For $p>p_{\mathrm{EM}}^{*}$, the minimum of $S_{A A^{\prime}}-S_{B A^{\prime}}$ is obtained at $q=q_{B A^{\prime}}^{*}(p)$.

Therefore, we have found $E_{M}=S_{A}$ for $p \leq p_{\mathrm{EM}}^{*}$, and $E_{M}=\frac{1}{2}\left(S_{A}+S_{B}+S_{A A^{*}}-S_{B A^{*}}\right)=\frac{1}{2}\left(S_{A}-S_{A^{*}}+S_{A A^{*}}\right)$ for $p>p_{\mathrm{EM}}^{*}$. In the latter case, the size of $A^{*}$ is given by $q_{B A^{\prime}}^{*}(p)$, and $S_{A A^{*}}$ is in the phase (A2) and $S_{B A^{*}}$ is at the phase transition point $(\mathrm{B} 1)=(\mathrm{B} 2)$. We may compute $S_{A^{*}}$ as $S_{A^{*}}=\frac{1}{2}\left(S_{A}+S_{A B}-S_{B}\right)$ from the equality condition $(\mathrm{B} 1)=(\mathrm{B} 2)$.

After all, we obtain $E_{M}$ in the Araki-Lieb transition as

$$
\begin{aligned}
& E_{M}(A: B) \\
& = \begin{cases}S_{A} & \left(p<p_{\mathrm{EM}}^{*}\right) \\
\frac{1}{2}\left[\frac{1}{2} I(A: B)+S_{A A^{*}}\left(p, q_{B A^{\prime}}^{*}(p)\right)\right] & \left(p>p_{\mathrm{EM}}^{*}\right)\end{cases}
\end{aligned}
$$

where $S_{A A^{*}} \equiv S_{A A^{*}}\left(p, q_{B A^{\prime}}^{*}(p)\right)$ denotes a contribution from the geodesics between $\partial A$ and $\partial A^{*}$,

$$
S_{A A^{*}}\left(p, q_{B A^{\prime}}^{*}(p)\right)=\frac{c}{3} \log \left(\frac{(1-p)\left(1+6 p+p^{2}\right)}{4 \sqrt{p} \epsilon}\right) .
$$

This result (51) confirms the shortcut formula (38). Note that $\frac{1}{2} I(A: B)=S_{A}$ for $p<p_{\mathrm{MI}}^{*}$ and that the balanced

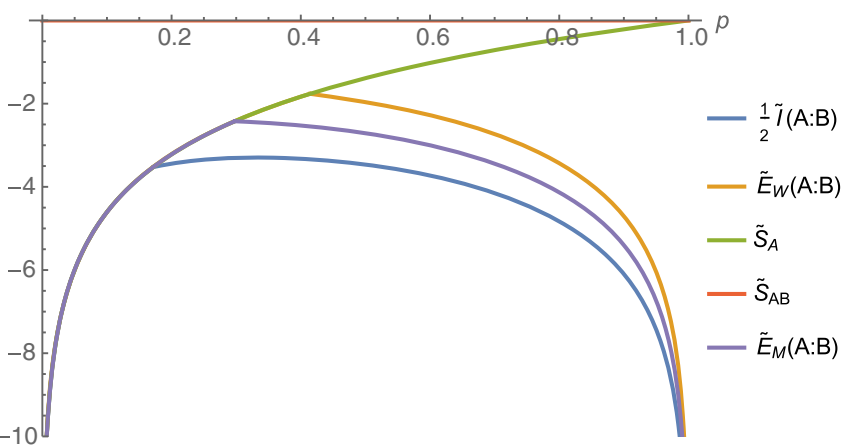

FIG. 11. The $E_{M}$ for the Araki-Lieb transition (normalized by subtracting $S_{A B}$ ).

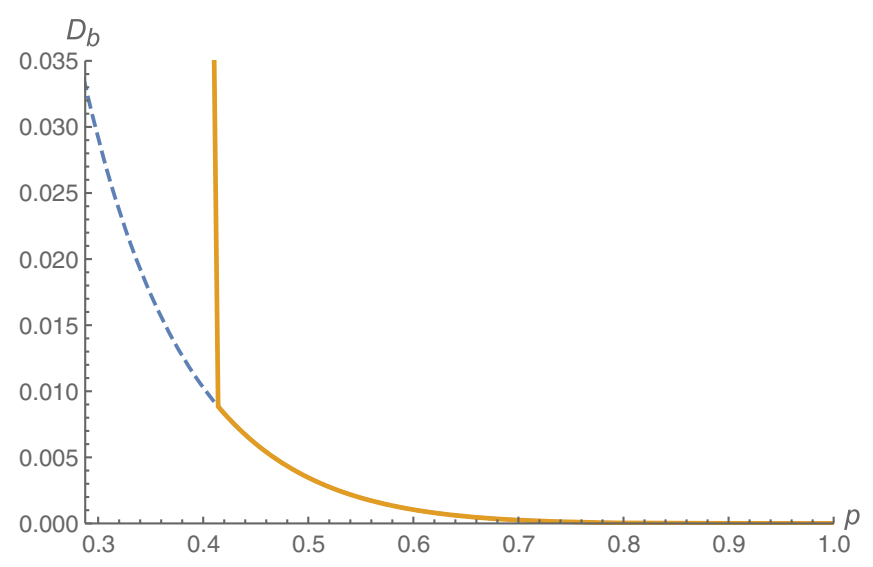

FIG. 12. The deviation $D_{b}$ for the Araki-Lieb transition (yellow solid line), and the $D_{b}$ replaced $E_{W}$ with the nonoptimal configuration in the Fig. 3 (blue dashed line).

optimal partition for $p \in\left(p_{\mathrm{MI}}^{*}, p_{\mathrm{EM}}^{*}\right)$ is given by $A^{\prime}$ of the size $q=q_{B A^{\prime}}^{*}(p)$, not the trivial partition (though it is also optimal). The balancing condition $I\left(A: M^{*}\right)=I\left(B: M^{*}\right)$ generically corresponds to the condition $(\mathrm{B} 1)=(\mathrm{B} 2)$. The deviation (39) is given by

$D_{b}(p)=S_{A A^{*}}\left(p, q_{B A^{\prime}}^{*}\right)-E_{W}(p)=\frac{c}{3} \log \frac{1+6 p+p^{2}}{4 \sqrt{p}(1+p)}$.

The plots of $E_{M}$ and $D_{b}$ are given in Figs. 11 and 12 .

In particular, the strict inequality $p_{\mathrm{MI}}^{*}<p_{\mathrm{EM}}^{*}$ indicates that $E_{M}$ must be strictly greater than the axiomatic entanglement measures for $p \in\left(p_{\mathrm{MI}}^{*}, p_{\mathrm{EM}}^{*}\right)$, based on the same logic as the EWCS. One can also confirm that $E_{M}$ exhibits the same kind of phase transition in the global BTZ black hole.

\section{DISCUSSION}

We have introduced a series of possible holographic duals to the optimized correlation measures. The crucial 
assumption for the equivalence was that the geometrical extensions are enough to achieve their minimum in holographic CFTs. They demonstrate many properties that are completely consistent with the original informationtheoretic measures.

We showed that the EWCS and the EWMI can be larger than the wide class of entanglement measures, while the holographic mutual information is not necessarily. This implies that the EWCS and the EWMI should be more sensitive to classical correlations than the holographic mutual information. Note that both the EWCS and the EWMI satisfy the strong superaddivity, which is a weaker property of quantum entanglement than the monogamy relation (since the latter induces the former). In addition, the EOP or the reflected entropy is supposed to be more sensitive to classical correlations than the mutual information $[32,40]$. It will be interesting future work to investigate a role of classical correlation in holographic CFTs.

There is a caveat that all of our discussions are restricted to the leading order $O\left(N^{2}\right)$. In particular, the Araki-Lieb transition relies on the property of the holographic entanglement entropy at this order. If one includes quantum corrections from bulk entanglement entropy at $O\left(N^{0}\right)$ $[73,74]$, the rigorous relation will be violated. For instance, the structure of state (15) is not robust against small correction to the exact saturation [75]. The saturation of the EWCS or the EWMI should also be found only in the large $N$ limit. We expect, however, that our conclusion itself still survives: the EWCS and the EWMI with some appropriate quantum corrections will still capture classical correlations.

The bit thread formalism [76] has been cooperative with these holographic optimized correlation measures. The bit threads for the bipartite EWCS were discussed in [77-80] and generalized to the multipartite EWCS [80]. It is interesting to seek a bit thread formalism for $E_{M}$ as well. Also, a multipartite generalization of $E_{M}$ would provide us a new tool to probe a specific aspect of the holographic correlations.

\section{ACKNOWLEDGMENTS}

We are grateful to Arpan Bhattacharyya, Souvik Dutta, Jonathan Harper, Masamichi Miyaji, Yoshifumi Nakata, Tadashi Takayanagi, and Yang Zhou for valuable comments on a draft of the present paper. We thank Jesse C. Cresswell and Massimiliano Rota for a lot of stimulating discussions that essentially motivated this work. We are grateful to "Quantum Information and String Theory 2019" at the Yukawa Institute for Theoretical Physics in Kyoto University where this work was initiated. We also acknowledge "Gravity - new perspectives from strings and higher dimensions 2019" at the Centro de Ciencias de Benasque Pedro Pascual, where this work was completed. K. U. is supported by Grant-in-Aid for JSPS fellows Grant No. $18 J 22888$.
Note.-We recently became aware that an independent work [68] that partially overlaps with the present paper will appear soon.

\section{APPENDIX: THE MULTIPARTITE GENERALIZATION}

In this Appendix, we complement some missing pieces in the previous study [22] of the multipartite generalization of the mutual information, the EOP, and the squashed entanglement as well as their holographic duals. The mutual information $I(A: B)$ has various multipartite generalizations. One of them is called the total correlation defined by

$$
\begin{aligned}
T_{n}\left(A_{1}: \cdots: A_{n}\right):= & S\left(\rho_{A} \| \rho_{A_{1}} \otimes \cdots \otimes \rho_{A_{n}}\right) \\
= & \sum_{i=1}^{n} S_{A_{i}}-S_{A} \\
= & I\left(A_{1}: A_{2}\right)+I\left(A_{1} A_{2}: A_{3}\right)+\cdots \\
& +I\left(A_{1} \cdots A_{n-1}: A_{n}\right),
\end{aligned}
$$

where $S(\rho \| \sigma)=\operatorname{Tr} \rho(\log \rho-\log \sigma)$ is the relative entropy. There is another generalization called the dual total correlation,

$$
\begin{aligned}
D_{n}\left(A_{1}: \cdots: A_{n}\right):= & S_{A_{1} \cdots A_{n}}-\sum_{i=1}^{n} S\left(A_{i} \mid A_{1} \cdots A_{n}\right) \\
= & I\left(A_{1}: A_{2} \cdots A_{n}\right)+I\left(A_{2}: A_{3} \cdots A_{n} \mid A_{1}\right) \\
& +\cdots+I\left(A_{n-1}: A_{n} \mid A_{1} \cdots A_{n-2}\right),
\end{aligned}
$$

where $S(A \mid B)=S_{A B}-S_{B}$ is the conditional entropy and i

$\cdots$ denotes the exclusion of $A_{i}$. The $T_{n}$ and $D_{n}$ are monotonically nonincreasing under strict local operations, vanish if and only if the state is totally decoupled, and $T_{n}=D_{n}=\sum_{i=1}^{n} S_{A_{i}}$ if the state is pure.

A multipartite generalization of the EOP [22,24] and the squashed entanglement $[81,82]$ are given as follows:

$$
\begin{gathered}
E_{P}\left(A_{1}: \cdots: A_{n}\right)=\frac{1}{2} \min _{|\psi\rangle_{A_{1} A_{1}^{\prime} \cdots A_{n} A_{n}^{\prime}}} T_{n}\left(A_{1} A_{1}^{\prime}: \cdots: A_{n} A_{n}^{\prime}\right) . \\
E_{s q}\left(A_{1}: \cdots: A_{n}\right)=\frac{1}{2} \min _{\rho_{A_{1} \cdots A_{n} E}} T_{n}\left(A_{1}: \cdots: A_{n} \mid E\right),
\end{gathered}
$$

where $T_{n}\left(A_{1}: \cdots: A_{n} \mid E\right)=I\left(A_{1}: A_{2} \mid E\right)+I\left(A_{1} A_{2}: A_{3} \mid E\right)+\cdots+$ $I\left(A_{1} \cdots A_{n-1}: A_{n} \mid E\right)$. The multipartite EOP is monotonically nonincreasing under strict local operations. The holographic dual of the multipartite EOP was proposed as the multipartite EWCS [22]. 
We can generalize the discussion of the holographic dual of the bipartite squashed entanglement as follows. The multipartite squashed entanglement can be written as

$$
E_{s q}=\frac{1}{2} T_{n}\left(A_{1}: \cdots: A_{n}\right)+\frac{1}{2} \min _{\rho_{A_{1} \cdots A_{n} E}} Q_{n}(A: E),
$$

where we define $Q_{n}(A ; E):=I\left(A_{1} \cdots A_{n}: E\right)-\sum_{i=1}^{n} I\left(A_{i}: E\right)$. This can be both positive and negative in the generic quantum system. In holography, however, the monogamy of mutual information implies $Q_{n} \geq 0$. For $n=2$, it reproduces the nonpositivity of tripartite information $Q_{2}(A B ; E)=$ $I(A B: E)-I(A: E)-I(B: E)=-I_{3}(A: B: E) \geq 0$. It again results in a conjecture that holographic multipartite squashed entanglement is equivalent to half of the total correlation,

$$
E_{s q}\left(A_{1}: \cdots: A_{n}\right)=\frac{1}{2} T_{n}\left(A_{1}: \cdots: A_{n}\right) .
$$

For the latter convenience, we introduce two nonnegative quantities for $n \geq 3$,

$$
X_{n}:=\frac{(n-1) T_{n}-D_{n}}{n-2}, \quad Y_{n}:=\frac{(n-1) D_{n}-T_{n}}{n-2} .
$$

They are normalized so that $X_{n}=Y_{n}=\sum_{i=1}^{n} S_{A_{i}}$ holds for pure states. They are positive semidefinite as it is clear from the following expressions,

$$
\begin{aligned}
& X_{n}\left(A_{1}: \cdots: A_{n}\right)=\frac{1}{n-2} \sum_{i=1}^{n} T_{n-1}\left(A_{1}: \stackrel{i}{\cdots}: A_{n}\right), \\
& Y_{n}\left(A_{1}: \cdots: A_{n}\right)=\frac{1}{n-2} \sum_{i=1}^{n} D_{n-1}\left(A_{1}: \stackrel{i}{\cdots}: A_{n} \mid A_{i}\right),
\end{aligned}
$$

where $\quad D_{n}\left(A_{1}: \cdots: A_{n} \mid E\right)=I\left(A_{1}: A_{2} \cdots A_{n} \mid E\right)+I\left(A_{2}: A_{3} \cdots\right.$ $\left.A_{n} \mid A_{1} E\right)+\cdots+I\left(A_{n-1}: A_{n} \mid A_{1} \cdots A_{n-2} E\right) . X_{n}$ is monotonically nonincreasing under strict local operations, while the $Y_{n}$ is not necessarily. Both $X_{n}$ and $Y_{n}$ are not faithful; i.e., there exists a state that is not decoupled $\rho_{A} \neq \rho_{A_{1}} \otimes \cdots \otimes \rho_{A_{n}}$ but $X_{n}=0$ or $Y_{n}=0$. Thus we do not consider each of them as a good correlation measure. Note a balance equation,

$$
T_{n}+D_{n}=X_{n}+Y_{n}=\sum_{i=1}^{n} I\left(A_{1}: \cdots: A_{n}\right)
$$

For holographic states, the monogamy of mutual information leads to a generic ordering,

$$
X_{n} \leq T_{n} \leq D_{n} \leq Y_{n} .
$$

Indeed, $T_{n} \leq D_{n}$ follows from the monogamy of mutual information,

$$
\begin{aligned}
D\left(A_{1}: \cdots: A_{n}\right)= & I\left(A_{1}: A_{2} \cdots A_{n}\right)+I\left(A_{2}: A_{3} \cdots A_{n} \mid A_{1}\right) \\
& +\cdots+I\left(A_{n-1}: A_{n} \mid A_{1} \cdots A_{n-2}\right) \\
\geq & I\left(A_{1}: A_{2} \cdots A_{n}\right)+I\left(A_{2}: A_{3} \cdots A_{n}\right) \\
& +\cdots+I\left(A_{n-1}: A_{n}\right) \\
= & T\left(A_{1}: \cdots: A_{n}\right) .
\end{aligned}
$$

Then $X_{n} \leq T_{n}$ and $D_{n} \leq Y_{n}$ are obvious by their definition.

Now we present some lower bounds on the multipartite EOP, which generalizes and complements the inequalities proven in [22]. The multipartite EOP is bounded from below by half of any multipartite correlation measure $\Theta$, which satisfies (i) $\Theta=\sum_{i=1}^{n} S_{A_{i}}$ for pure $n$-partite states, and (ii) is nonincreasing under strict local operations,

$$
E_{P} \geq \frac{1}{2} \Theta
$$

It is obvious from the definition (A6) following the same logic as in [22]. Here $T_{n}, D_{n}$, and $X_{n}$ satisfy both conditions, but $Y_{n}$ does not satisfy (ii). Thus, we get three inequalities for generic multipartite states

$$
E_{P} \geq \frac{1}{2} \max \left\{X_{n}, T_{n}, D_{n}\right\} .
$$

The lower bounds by $T_{n}$ and $X_{3}$ were proven in [22], and the above inequality gives $n$-partite generalization for $X_{n}$. On the other hand, the bound by $D_{n}$ is totally new. Interestingly, $D_{n}$ gives a stricter lower bound on $E_{P}$ than $T_{n}$ in holography by the ordering (A14). One can check that $E_{W} \geq \frac{1}{2} D_{n}$ always holds, while $E_{W} \geq \frac{1}{2} Y_{n}$ is not true in general.
[1] J. M. Maldacena, The Large N limit of superconformal field theories and supergravity, Int. J. Theor. Phys. 38, 1113 (1999); Adv. Theor. Math. Phys. 2, 231 (1998).

[2] S. S. Gubser, I. R. Klebanov, and A. M. Polyakov, Gauge theory correlators from noncritical string theory, Phys. Lett. B 428, 105 (1998).

[3] E. Witten, Anti-de Sitter space and holography, Adv. Theor. Math. Phys. 2, 253 (1998).
[4] J. M. Maldacena, Eternal black holes in anti-de Sitter, J. High Energy Phys. 04 (2003) 021.

[5] M. Van Raamsdonk, Building up spacetime with quantum entanglement, Gen. Relativ. Gravit. 42, 2323 (2010); Int. J. Mod. Phys. D 19, 2429 (2010).

[6] J. Maldacena and L. Susskind, Cool horizons for entangled black holes, Fortschr. Phys. 61, 781 (2013). 
[7] T. Takayanagi, Holographic Spacetimes as Quantum Circuits of Path-Integrations, J. High Energy Phys. 12 (2018) 048.

[8] M. Van Raamsdonk, Building up spacetime with quantum entanglement II: It from BC-bit, arXiv:1809.01197.

[9] S. Ryu and T. Takayanagi, Holographic Derivation of Entanglement Entropy from AdS/CFT, Phys. Rev. Lett. 96, 181602 (2006).

[10] A. Lewkowycz and J. Maldacena, Generalized gravitational entropy, J. High Energy Phys. 08 (2013) 090.

[11] V.E. Hubeny, M. Rangamani, and T. Takayanagi, A covariant holographic entanglement entropy proposal, J. High Energy Phys. 07 (2007) 062.

[12] X. Dong, A. Lewkowycz, and M. Rangamani, Deriving covariant holographic entanglement, J. High Energy Phys. 11 (2016) 028.

[13] M. J. Donald, M. Horodecki, and O. Rudolph, The uniqueness theorem for entanglement measures, J. Math. Phys. (N.Y.) 43, 4252 (2002).

[14] K. Umemoto and T. Takayanagi, Entanglement of purification through holographic duality, Nat. Phys. 14, 573 (2018).

[15] P. Nguyen, T. Devakul, M. G. Halbasch, M. P. Zaletel, and B. Swingle, Entanglement of purification: from spin chains to holography, J. High Energy Phys. 01 (2018) 098.

[16] A. C. Wall, Maximin surfaces, and the strong subadditivity of the covariant holographic entanglement entropy, Classical Quantum Gravity 31, 225007 (2014).

[17] B. Czech, J. L. Karczmarek, F. Nogueira, and M. Van Raamsdonk, The gravity dual of a density matrix, Classical Quantum Gravity 29, 155009 (2012).

[18] M. Headrick, V. E. Hubeny, A. Lawrence, and M. Rangamani, Causality \& holographic entanglement entropy, J. High Energy Phys. 12 (2014) 162.

[19] B. M Terhal, M. Horodecki, D. W. Leung, and D. P. DiVincenzo, The entanglement of purification, J. Math. Phys. (N.Y.) 43, 4286 (2002).

[20] B. Swingle, Entanglement renormalization and holography, Phys. Rev. D 86, 065007 (2012).

[21] M. Miyaji and T.Takayanagi, Surface/state correspondence as a generalized holography. Prog. Theor. Exp. Phys. 2015, 073 B03 (2015).

[22] K. Umemoto and Y. Zhou, Entanglement of purification for multipartite states and its holographic dual, J. High Energy Phys. 10 (2018) 152.

[23] N. Bao and I. F. Halpern, Holographic inequalities and entanglement of purification, J. High Energy Phys. 03 (2018) 006.

[24] N. Bao and I. F. Halpern, Conditional and multipartite entanglements of purification and holography, Phys. Rev. D 99, 046010 (2019).

[25] H. Hirai, K. Tamaoka, and T. Yokoya, Towards entanglement of purification for conformal field theories, Prog. Theor. Exp. Phys. 2018, 063 B03 (2018).

[26] R. Espíndola, A. Guijosa, and J. F. Pedraza, Entanglement wedge reconstruction and entanglement of purification, Eur. Phys. J. C 78, 646 (2018).

[27] N. Bao, A. Chatwin-Davies, and G. N. Remmen, Entanglement of purification and multiboundary wormhole geometries, J. High Energy Phys. 02 (2019) 110.
[28] R.-Q. Yang, C.-Y. Zhang, and W.-M. Li, Holographic entanglement of purification for thermofield double states and thermal quench, J. High Energy Phys. 01 (2019) 114.

[29] P. Caputa, M. Miyaji, T. Takayanagi, and K. Umemoto, Holographic Entanglement of Purification from Conformal Field Theories, Phys. Rev. Lett. 122, 111601 (2019).

[30] P. Liu, Y. Ling, C. Niu, and J.-P. Wu, Entanglement of purification in holographic systems, J. High Energy Phys. 09 (2019) 071.

[31] W.-Z. Guo, Entanglement of purification and projective measurement in conformal field theories, Phys. Lett. B797, 134934 (2019).

[32] A. Bhattacharyya, A. Jahn, T. Takayanagi, and K. Umemoto, Entanglement of Purification in Many Body Systems and Symmetry Breaking, Phys. Rev. Lett. 122, 201601 (2019).

[33] M. Ghodrati, X.-M. Kuang, B. Wang, C.-Y. Zhang, and Y.-T. Zhou, The connection between holographic entanglement and complexity of purification, J. High Energy Phys. 09 (2019) 009.

[34] A. Prudenziati, A geodesic Witten diagram description of holographic entanglement entropy and its quantum corrections, J. High Energy Phys. 06 (2019) 059.

[35] N. Bao, G. Penington, J. Sorce, and A. C. Wall, Holographic tensor networks in full AdS/CFT, arXiv:1902.10157.

[36] K. B. Velni, M. R. M. Mozaffar, and M. H. Vahidinia, Some aspects of entanglement wedge cross-section, J. High Energy Phys. 05 (2019) 200.

[37] T. Ota, Comments on holographic entanglements in cutoff AdS, arXiv:1904.06930.

[38] N. Jokela and A. Pönni, Notes on entanglement wedge cross sections, J. High Energy Phys. 07 (2019) 087.

[39] W.-Z. Guo, Entanglement of purification and disentanglement in CFTs, J. High Energy Phys. 09 (2019) 080.

[40] Y. Kusuki and K. Tamaoka, Dynamics of entanglement wedge cross section from conformal field theories, arXiv:1907.06646.

[41] J. Kudler-Flam and S. Ryu, Entanglement negativity and minimal entanglement wedge cross sections in holographic theories, Phys. Rev. D 99, 106014 (2019).

[42] J. Kudler-Flam, I. MacCormack, and S. Ryu, Holographic entanglement contour, bit threads, and the entanglement tsunami, J. Phys. A 52, 325401 (2019).

[43] J. Kudler-Flam, M. Nozaki, S. Ryu, and M. T. Tan, Quantum vs. classical information: operator negativity as a probe of scrambling, arXiv:1906.07639.

[44] Y. Kusuki, J. Kudler-Flam, and S. Ryu, Derivation of Holographic Negativity in $\mathrm{AdS}_{3} / \mathrm{CFT}_{2}$, Phys. Rev. Lett. 123, 131603 (2019).

[45] K. Tamaoka, Entanglement Wedge Cross Section from the Dual Density Matrix., Phys. Rev. Lett. 122, 141601 (2019).

[46] S. Dutta and T. Faulkner, A canonical purification for the entanglement wedge cross-section, arXiv:1905.00577.

[47] P. Hayden, M. Headrick, and A. Maloney, Holographic mutual information is monogamous, Phys. Rev. D 87, 046003 (2013).

[48] R. Horodecki, P. Horodecki, M. Horodecki, and K. Horodecki, Quantum entanglement, Rev. Mod. Phys. 81, 865 (2009). 
[49] V. E. Hubeny, H. Maxfield, M. Rangamani, and E. Tonni, Holographic entanglement plateaux, J. High Energy Phys. 08 (2013) 092.

[50] M. Headrick, General properties of holographic entanglement entropy, J. High Energy Phys. 03 (2014) 085.

[51] L Henderson and V. Vedral, Classical, quantum and total correlations. J. Phys. A 34, 6899 (2001).

[52] H. Ollivier and W. H. Zurek, Quantum Discord: A Measure of the Quantumness of Correlations, Phys. Rev. Lett. 88, 017901 (2001).

[53] M. Christandl and A. Winter, "Squashed entanglement": An additive entanglement measure, J. Math. Phys. (N.Y.) 45, 829 (2004).

[54] D. Yang, M. Horodecki, and Z. D. Wang, An Additive and Operational Entanglement Measure: Conditional Entanglement of Mutual Information, Phys. Rev. Lett. 101, 140501 (2008).

[55] J. Levin and G. Smith, Optimized measures of bipartite quantum correlation, arXiv:1906.10150.

[56] S. Bagchi and A. K. Pati, Monogamy, polygamy, and other properties of entanglement of Purification, Phys. Rev. A 91, 042323 (2015).

[57] C. H. Bennett, D. P. DiVincenzo, J. A. Smolin, and W. K. Wootters, Mixed-state entanglement and quantum error correction, Phys. Rev. A 54, 3824 (1996).

[58] E. M. Rains, Rigorous treatment of distillable entanglement, Phys. Rev. A 60, 173 (1999).

[59] R. R. Tucci, Entanglement of distillation and conditional mutual information, arXiv:quant-ph/0202144.

[60] V. Vedral, M. B. Plenio, M. A. Rippin, and P. L. Knight, Quantifying Entanglement, Phys. Rev. Lett. 78, 2275 (1997).

[61] P. M. Hayden, M. Horodecki, and B. M. Terhal, The asymptotic entanglement cost of preparing a quantum state, J. Phys. A 34, 6891 (2001).

[62] N. Li and S. Luo, Total versus quantum correlations in quantum states, Phys. Rev. A 76, 032327 (2007).

[63] Z. Xi, X.-M. Lu, X. Wang, and Y. Li, Necessary and sufficient condition for saturating the upper bound of quantum discord, Phys. Rev. A 85, 032109 (2012).

[64] L. Zhang and J. Wu, On conjectures of classical and quantum correlations in bipartite States, J. Phys. A 45, 025301 (2012).

[65] M. Christandl and A. Winter, Uncertainty, monogamy, and locking of quantum correlations, IEEE Trans. Inf. Theory 51, 3159 (2005).

[66] A. Uhlmann, The "transition probability" in the state space of a *-algebra, Rep. Math. Phys. 9, 273 (1976).
[67] M. Koashi and A. Winter, Monogamy of quantum entanglement and other correlations, Phys. Rev. A 69, 022309 (2004).

[68] J. Levin, O. DeWolfe, and G. Smith, Correlation measures and distillable entanglement in ads/cft, arXiv:1909.04727.

[69] N. Bao, S. Nezami, H. Ooguri, B. Stoica, J. Sully, and M. Walter, The holographic entropy cone, J. High Energy Phys. 09 (2015) 130.

[70] M. Rota and S. J. Weinberg, New constraints for holographic entropy from maximin: A no-go theorem, Phys. Rev. D 97, 086013 (2018).

[71] V. E. Hubeny, M. Rangamani, and M. Rota, The holographic entropy arrangement, Fortschr. Phys. 67, 1900011 (2019).

[72] S. H. Cuenca, Holographic entropy cone for five regions, Phys. Rev. D 100, 026004 (2019).

[73] T. Faulkner, A. Lewkowycz, and J. Maldacena, Quantum corrections to holographic entanglement entropy, J. High Energy Phys. 11 (2013) 074.

[74] T. Barrella, X. Dong, S. A. Hartnoll, and V. L. Martin, Holographic entanglement beyond classical gravity, J. High Energy Phys. 09 (2013) 109.

[75] P. Hayden, R. Jozsa, D. Petz, and A. Winter, Structure of states which satisfy strong subadditivity of quantum entropy with equality, Commun. Math. Phys. 246, 359 (2004).

[76] M. Freedman and M. Headrick, Bit threads and holographic entanglement, Commun. Math. Phys. 352, 407 (2017).

[77] C. A. Agón, J. De Boer, and J. F. Pedraza, Geometric aspects of holographic bit threads, J. High Energy Phys. 05 (2019) 075 .

[78] D.-H. Du, C.-B. Chen, and F.-W. Shu, Bit threads and holographic entanglement of purification, J. High Energy Phys. 08 (2019) 140.

[79] N. Bao, A. Chatwin-Davies, J. Pollack, and G. N. Remmen, Towards a bit threads derivation of holographic entanglement of purification, J. High Energy Phys. 07 (2019) 152.

[80] J. Harper and M. Headrick, Bit threads and holographic entanglement of purification, J. High Energy Phys. 08 (2019) 101.

[81] D. Yang, K. Horodecki, M. Horodecki, P. Horodecki, J. Oppenheim, and W. Song, Squashed entanglement for multipartite states and entanglement measures based on the mixed convex roof, IEEE Trans. Inf. Theory 55, 3375 (2009).

[82] D. Avis, P. Hayden, and I. Savov, Distributed compression and multiparty squashed entanglement, J. Phys. A 41, 115301 (2008). 